\title{
Recent Changes in Tuberculosis Guidelines: What's New for Ocular Tuberculosis
}

\author{
Amber Kumar ${ }^{2}$, Priyanka ${ }^{1 *}$ and Mahesh Maheshwari ${ }^{3}$ \\ ${ }^{1}$ Department of Ophthalmology, AIIMS, Bhopal, India \\ ${ }^{2}$ Assistant Professor, Department of Pediatrics, AIIMS, Bhopal, India \\ ${ }^{3}$ Associate Professor, Department of Pediatrics, AIIMS, Bhopal, India
}

*Corresponding author: Dr Priyanka, Department of Ophthalmology, AIIMS, Bhopal, India

\begin{abstract}
Ocular TB can cause moderate to severe visual impairment in up to $40 \%$ of affected eyes. Delay in diagnosis and treatment can result in chronic inflammation and loss of vision. The pathogenesis of ocular tuberculosis is still not clear, whether it result from a direct mycobacterial infection or a hypersensitivity response to mycobacterium tuberculosis. The choroidal nodules likely link from direct hematogenous infection while the vasculitis and choroiditis are probably the result of immune hypersensitivity. Prevalence of tuberculosis as an etiology of uveitis range from 5-10\% in endemic areas. Tuberculous uveitis inevitably leads to blindness if not properly diagnosed and treated. The aim of this paper is to review the presentation, diagnosis and management of tuberculous uveitis and to highlight the recent changes in the tuberculosis guidelines. Indian extrapulmonary tuberculosis (EPTB) guidelines were developed under the auspices of Central TB Division and Directorate of Health Services, Ministry of Health and Family Welfare, Government of India in 2016, with the objective of providing guidance on uniform, evidence-informed practices for suspecting, diagnosing and managing EPTB.
\end{abstract}

Keywords: ATT; Diagnosis; Ocular Tuberculosis; TST; Uveitis

\section{Introduction}

Tuberculosis (TB) is a global health problem. It is a chronic, granulomatous infection caused by Mycobacterium tuberculosis. This acid-fast bacillus usually affects the lungs but can affect any organ of body except teeth and hairs. Tuberculosis can be considered in the differential diagnosis of any type of intraocular inflammation. Because of the lack of standardized diagnostic criteria for tuberculous uveitis, and the difficulty of confirming the diagnosis by laboratory methods, epidemiologic data for tuberculous uveitis are unreliable. Incidence of tubercular uveitis in developed countries is less than developing countries. In India, where pulmonary TB is endemic, TB was the cause of uveitis in 5.6$10.1 \%$ of the cases [1]. The aim of this review article is to focus on the diagnostic criteria and the management of tuberculous uveitis and to highlight the key changes as recommended in INDEX TB guidelines.

\section{Pathogenesis}

Ocular TB is either primary, in which the eye is the primary port of entry of the mycobacterium into the body, or secondary, as a result of seeding by hematogenous spread from a distant site. Primary disease usually involves eyelid, conjunctival, corneal, and scleral lesions, but are rare entities, while the secondary tuberculous disease involves uveal tract, retina, and optic nerve. Inflammation of the uveal tract is the most common eye manifestation of the disease, due to its high blood supply [2].

\section{Diagnosis}

Most patients with ocular involvement have no history of pulmonary or other systemic forms making a definitive diagnosis difficult [3-4]. Therefore, tuberculous uveitis is frequently under diagnosed and the disease is recognizable after a very long diagnostic delay [5]. The absence of clinically evident pulmonary TB does not rule out the possibility of ocular TB, as about $60 \%$ of patients with extra pulmonary TB have no evidence of pulmonary TB [6].

Ocular infection with M. tuberculosis is uncommon, but prevalence estimates may not be reliable because of diagnostic difficulties. Incidence of TB as a cause among patients presenting 
with uveitis has been reported at $10.1 \%$ in north India (Singh, 2004), but much lower in south India at 0.6\% (Biswas, 1996-1997). This discrepancy may be due to several factors, including access to ophthalmology services, evolution of diagnostic criteria, description of new diagnostic entities and improvement in diagnostic tools [7].

There is always conflict between ophthalmologists and physicians for instituting anti-tubercular treatment (ATT) due to lack of gold standard criteria to diagnose ocular TB. Usually the patients referred to a Pulmonologist due to a suspicion of tuberculous uveitis will lack any clinical signs of a systemic disease on routine investigations and finally the decision to treat will lie on the ophthalmologist. The current practice at most of the centre is to make a presumptive diagnosis with positive TB skin test (TST), positive interferon gamma release assays (IGRA), and lesions on imaging of the chest (chest x-ray/ chest CT scan). Either resolution of uveitis or nonrecurrence of the uveitis after treatment completion of TB supports the diagnosis [7]. Before proceeding for full antituberculous treatment treating physician should always keep in mind the potential dangers of the therapeutic management.

There is a scarcity of data from clinical trials in EPTB and most of the information regarding diagnosis and management is extrapolated from pulmonary TB. Indian EPTB guidelines were developed under the auspices of Central TB Division and Directorate of Health Services, Ministry of Health and Family Welfare, Government of India in 2016, with the objective of providing guidance on uniform, evidence-informed practices for suspecting, diagnosing and managing EPTB.

The following are the key points to remember in case of ocular tuberculosis

\section{Patients who require Ophthalmologist Review}

Patients with symptoms consistent with anterior, intermediate, posterior or pan-uveitis, including the following: Red eye, blurred vision, photophobia, irregular pupil, eye pain, floaters, photopsia.

\section{Investigations for Ocular TB}

All suspected cases should undergo comprehensive examination including complete blood count, erythrocyte sedimentation rate, liver enzyme panel, infectious disease screen (which included Venereal Disease Research Laboratory (VDRL) test for syphilis, urine microscopy, serum ACE level), chest x ray, mantoux test, Quantiferon Gold test, and HRCT Chest. Blood should be taken for QTB Gold before the performance of mantoux test. PCR assays for TB DNA can be performed in patients with severe anterior chamber inflammation to confirm TB.

Ocular Imaging: Fundus photography, fluorescein angiography or optical coherence tomography to assess extent or complications of disease, and to monitor response to treatment.

X-ray of Chest and CT of the Chest: To find out the evidence of past or present MTB infection. A CXR with evidence of pulmonary nodules, calcification or fibrosis in the hilar area or upper lobes was considered as a positive CXR finding.

HRCT chest with evidence of parenchymal cavitating lesions, nodular lesions with tree in bud appearance, lymph node with center necrosis, matting and pleural effusion, hilar and mediastinal lymphadenopathy were considered as active TB. HRCT chest with evidence of calcified granulomas predominantly in upper lobes, calcified mediastinal lymph nodes and apical scarring were considered as healed TB.

Immunological Test: Tuberculin Skin Testing (TST)/ Interferon-Gamma Release Assays (IGRA) (Quantiferon Gold): Can be performed in all suspected cases. The predictive value of TST varies depending on the incidence of $\mathrm{TB}$ in the population and local BCG vaccination policy. IGRA is based on gamma interferon production by $\mathrm{T}$ cells sensitized to MTB specific antigens. It is not influenced by BCG and most nontuberculous mycobacteria (NTM). Positivity of both tests only indicates exposure to tuberculosis/ latent infection, and it is not a indicator of active TB disease. While a positive result may support the diagnosis of $\mathrm{TB}$, a negative test cannot rule out TB.

CBNAAT Testing of Vitreous or Aqueous Specimens: Can be done in selected cases. The accuracy of Cartridge based nucleic acid amplification technique (CBNAAT) seems to vary significantly, but this is the only specific test that may identify ocular TB. Vitreous/ aqueous humour sampling by a trained person is recommended for CBNAAT testing.

Biopsy: Recommended in highly selected cases as biopsy of the structures of the eye is highly invasive and carries the risk of exacerbating visual loss. Specimens need histopathological examination with staining for acid-fast bacilli (AFB) and culture. So, should be performed in highly selected cases by the experienced persons.

Newer molecular methods like CBNAAT and line probe assay helps in diagnostic algorithm from any fluid/tissue samples for rapid detection and they also tells about drug sensitivity.

\section{Diagnostic Categories}

Can be divided into 4 categories

Presumptive Ocular TB: Patient with one of the following clinical presentations [7]:

I. Anterior Uveitis:

a. Granulomatous, mutton-fat keratic precipitates

b. Iris nodules

c. Broad-based posterior synechiae

d. Hypopyon may be observed

e. Vitritis 


\section{Intermediate uveitis}

III. Posterior Uveitis: Subretinal abscess, multifocal choroiditis, choroidal/disc granuloma, multifocal serpiginous choroiditis and retinal periphlebitis.

\section{Panuveitis}

V. Rarely, scleritis (anterior and posterior), interstitial and disciform keratitis Figure 1.

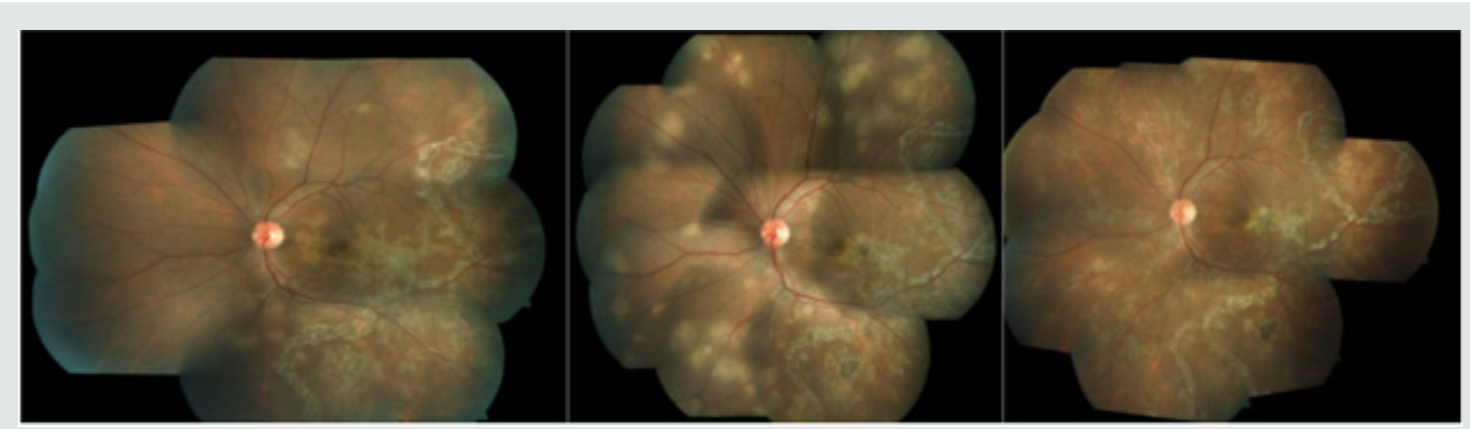

Figure 1: Fundus photo montage (LE) of a case of Tubercular Multifocal Choroiditis presented with active lesions threatening macula with laser marks (1), Lesions progressed at periphery on ATT at 1 month (possible Jarisch Herxheimer reaction) (2), Healed lesion at 3 months on addition of azathioprine along with steroid and continuation of ATT.

Note: Extraocular TB disease is often absent in ocular TB patients and patients do not usually have systemic symptoms of fever and weight loss. Of all the clinical manifestations, the presence of choroidal granulomas, occlusive retinal vasculitis, and multifocal serpiginoid choroiditis has been more strongly associated with presumed ocular tuberculosis. In particular, choroidal granulomas should raise a high index of suspicion and failure to recognize them could lead to devastating consequences.

\section{Possible Ocular TB:}

a. At least one clinical sign suggestive of ocular TB with exclusion of other aetiologies.

b. Documented exposure to TB (history of contact with sputum positive pulmonary TB case) and/or immunological evidence of TB infection (TST/Quantiferon gold test positivity).

Latent TB: Some of the patients had no evidence of active TB at another extraocular site. Demonstration of the presence of an immune response to TB antigens (Positive Mantoux or Quantiferon gold test) in the absence of evidence of active TB has been used to characterise the state of 'latent' TB. This term becomes confusing in the presence of active uveitis; this may be unrelated to the TB immune response, which therefore is truly latent. However, the uveitis may be an ophthalmic manifestation of TB, either directly or via a hypersensitivity mechanism. Despite the absence of other sites of active TB Ophthalmologist used to treat such cases as active TB.

\section{Clinically Diagnosed Ocular TB (Probable Ocular TB):}

a. At least one clinical sign suggestive of ocular TB with exclusion of other aetiologies.

b. Chest X-ray consistent with TB infection/Clinical evidence of extraocular TB/ microbiological confirmation from sputum or extraocular sites. c. Documented exposure to TB (history of contact with sputum positive pulmonary TB case) and/or immunological evidence of TB infection (TST/Quantiferon gold test positivity).

Bacteriologically Confirmed Ocular TB: Microbiological (smear/culture) or, histopathological confirmation of MTB from ocular fluids/tissues.

\section{Treatment of Ocular TB}

Treatment with ATT with or without other adjuvant therapy is recommended in all patients with possible ocular TB, clinically diagnosed ocular TB or bacteriologically confirmed ocular TB, with the aim to protect visual function, to control ocular inflammation and to prevent recurrence of inflammation.

First Line Treatment for Adults and Children with Ocular TB: Principle of treatment of TB has been now daily regimen with administration of daily fixed dose combination of first line ATT as per appropriate weight bands for both newly diagnosed and previously treated patients. (Both the Category II and III has been discontinued now).

2(HRZE) + 4 (HRE): Continue ethambutol in continuation phase too (new guideline) (H-Isoniazide, R-Rifampicin, Z-Pyrazinamide, E-Ethambutol).

Duration of extrapulmonary TB treatment (new guideline) The CP in both new and previously treated cases may be extended based on clinical decision of the treating physicians. Adjunctive treatments are corticosteroids (local or systemic) and other immunosuppressants.

I. Duration: Total treatment duration: 6 to 9 months.

II. Follow-up: Patients need regular monitoring during and after treatment with clinical evaluation (Slit-lamp biomicroscopy and Indirect Ophthalmoscopy). Fundus photo, fundus autofluorescence, fluorescine angiography and optical coherence tomography (OCT). 
Follow-up visits requires initially monthly, then 2-3 monthly (or more frequently as required) to monitor response to therapy with a minimum period of 6 months after completion of therapy.

Treatment Outcomes: In ocular TB defined differently than other pulmonary and extrapulmonary tuberculosis, as microbiological confirmation of TB is rarely possible in ocular tissues. Thus, treatment success or failure is primarily guided by the level of inflammation seen inside the eye. The main outcome measure was recurrence of inflammation after minimum six months of initiating treatment.

Remission: Inactive disease for at least 3 months after discontinuing all therapy based on the Standardization of Uveitis Nomenclature recommendations (Jabs, 2005).

Treatment Failure: No decrease in inflammation, or less than a two-step decrease in level of inflammation after 3 months of ATT.

Relapse: An increase in the level of inflammation after complete remission (at least two-step increase).

\section{Approach to Treatment Failure}

a. Work-up for other Etiology: Detailed ocular and systemic evaluation.

b. Look for Paradoxical Reaction: usually occurs within 2 months of starting ATT; responds to continuation or escalation of corticosteroid.

c. Rule out Drug Resistance: After ruling out first two points check history of contact with MDR TB patients and if facilities exist, consider ocular fluid sampling for molecular diagnosis of drug resistance.

d. Surgery: Is indicated in ocular TB in cases of complications of retinal vasculitis like retinal neovascularisation, vitreous haemorrhage or retinal detachment or complication of uveitis such as cataract and glaucoma.

Note: Introduction of Bedaquiline as a new drug. ATP synthase inhibitor specifically targets mycobacterial TB. Indicated in age more than 18 years [7].

\section{Conclusion}

Treatment with ATT with or without other adjuvant therapy is recommended in all patients with possible ocular TB, clinically diagnosed ocular TB or bacteriologically confirmed ocular TB. Principle of treatment of TB has been now daily regimen with administration of daily fixed dose combination of first line ATT for 6-9 months, as per appropriate weight bands for both newly diagnosed and previously treated patients, with continuation of ethambutol in continuation phase too. We recommend a high index of suspicion for tuberculous uveitis and a readiness to use ATT even where tuberculous aetiology has not been proven microbiologically but having clinical or radiologic findings of either active or latent TB infection and monitor for a response, in contrast to other forms of tuberculosis where this approach is not recommended. Randomized control trials are needed in the future but may be difficult to plan due to the low prevalence rate of ocular tuberculosis and until definitive diagnostic tests are available.

\section{References}

1. Singh R, Gupta V, Gupta A (2004) Pattern of uveitis in a referral eye clinic in north India. Indian J Ophthalmol 52: 121-125.

2. Helm CJ, Holland GN (1993) Ocular tuberculosis. Surv Ophthalmol 38: 229-256.

3. Rosen PH, Spalton DJ, Graham EM(1990) Intraocular tuberculosis. Eye 4: 486-492.

4. Cimino L, Herbort CP, Aldigeri R, Salvarani C, Boiardi L (2009) Tuberculous uveitis: A resurgent and underdiagnosed disease. Int Ophthalmol 29(2): 67-74.

5. Alvarez S, McCabe WR (1984) Extrapulmonary tuberculosis revisited: A review of experience at Boston City and other hospitals. Medicine (Baltimore) 63(1): 25-55.

6. Index TB Guidelines (2016) Guidelines on extra-pulmonary tuberculosis for India. World Health Organization.

7. Gupta V, Gupta A, Rao NA (2007) Intraocular tuberculosis-an update. Surv Ophthalmol 52(6): 561-587.

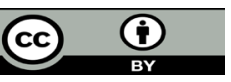

This work is licensed under Creative Commons Attribution 4.0 License

To Submit Your Article Click Here: Submit Article

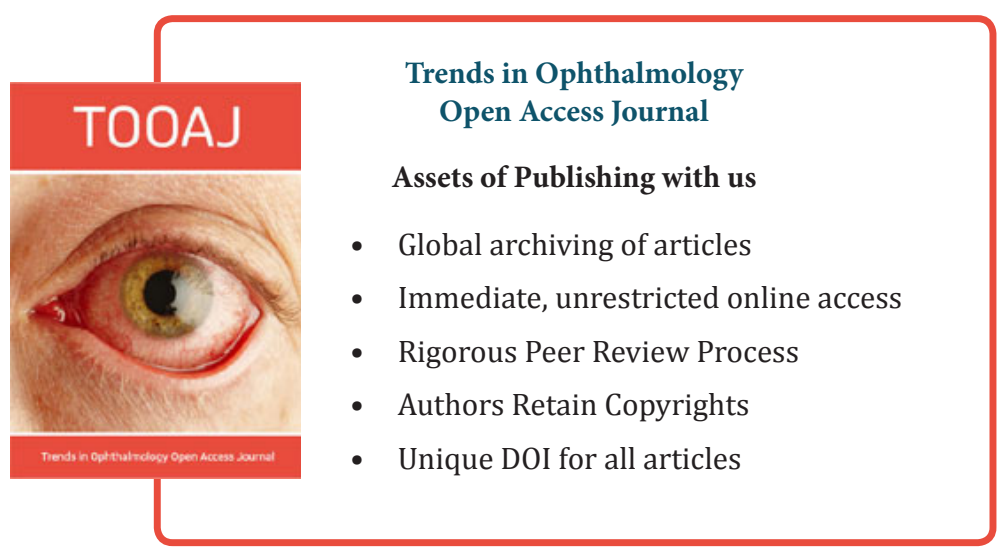

